NOTE

\title{
High sensitivity radiochromic film dose comparisons
}

\author{
Martin J Butson ${ }^{1,2}$, Peter K N Yu ${ }^{1}$, Tsang Cheung ${ }^{1}$ and Peter Metcalfe ${ }^{2}$ \\ ${ }^{1}$ Department of Physics and Materials Science, City University of Hong Kong, Kowloon Tong, \\ Hong Kong \\ ${ }^{2}$ Illawarra Cancer Care Centre, Department of Medical Physics, Crown Street, Wollongong, \\ NSW 2500, Australia \\ E-mail: butsonm@iahs.nsw.gov.au
}

Received 14 August 2002

Published 30 October 2002

Online at stacks.iop.org/PMB/47/N291

\begin{abstract}
This short note investigates the dose characteristics of a relatively new high sensitivity radiochromic film (Gafchromic HS) and compares dose and energy response to various Gafchromic film types and radiographic (EDR-2) film. The original MD-55-1 and two improved sensitivity films, MD-55-2 and HS film, were investigated for energy and dose response. Results show that the energy response of the new HS film is relatively the same as the original MD-55-1 and MD-55-2 films with a decrease in sensitivity at lower X-ray energies, with response decreasing down to approximately 0.64 (normalized to 1 for a $6 \mathrm{MV}$ beam) for a $28 \mathrm{keV}$ effective energy beam. This is compared to an over response of 9.2 at the same energy for EDR-2 film. The dose response at the maximum absorption peak was found to be approximately 3.8 and 1.9 times more sensitive than MD-55-1 and MD-55-2 films, respectively. At the absorption peak yielding the maximum optical density change, HS was found to be approximately 0.2 to 0.25 times the sensitivity of EDR-2.
\end{abstract}

\section{Introduction}

Due to its relatively low energy dependence compared to radiographic film, Gafchromic MD55-2 film has become a significant dosimetry tool in high-energy radiotherapy (Klassen et al 1997, Niroomand-Rad et al 1998, McLaughlin et al 1991). One of its shortcomings for use in both in-phantom and in vivo dosimetry, however, has been its low sensitivity, normally requiring large doses (5 Gy or more) to produce acceptable results. The introduction of a new film type, Gafchromic HS, is designed to increase dose response to high energy x-rays. This note investigates the dose and energy response properties of this new film and compares results to existing Gafchromic films and EDR-2 radiographic film which is used in IMRT applications. 


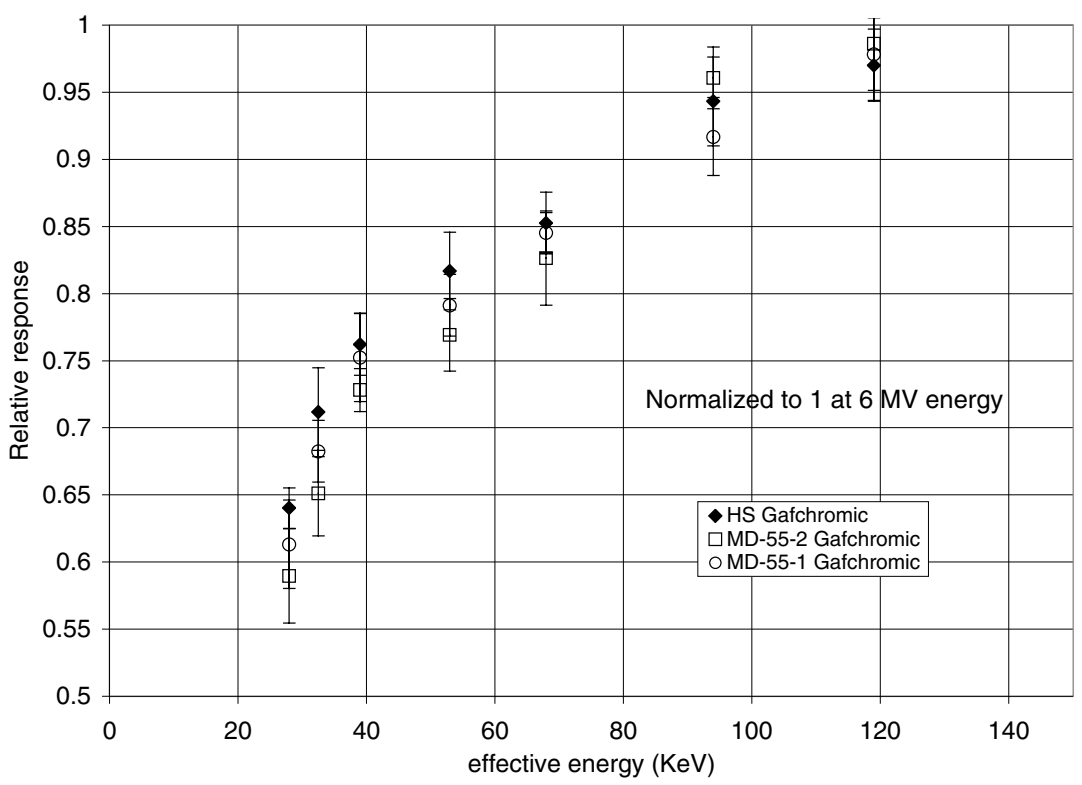

Figure 1. Energy response of HS, MD-55-2 and MD-55-1 Gafchromic film at superficial and orthovoltage energies.

\section{Materials and methods}

Gafchromic HS film consists of an active dosimetric layer sandwiched between two sheets of transparent $97 \mu \mathrm{m}$ polyester. The active layer has the same components as in MD-55-2 and is approximately $40 \mu \mathrm{m}$ thick. The new HS film also varies from its predecessor, MD-55-2, as it does not employ an adhesive layer, which was used in the MD-55 films to bond the two coated substrates. This feature provides a more flexible film.

Dose and energy dependence measurements were performed on a Varian 2100C accelerator at photon energies of $6 \mathrm{MV}$ and a Pantak 300DXT orthovoltage x-ray machine (Butson et al 1995) using beams with effective energies ranging from $28 \mathrm{keV}$ up to $123 \mathrm{keV}$. An RMI $30 \times 30 \mathrm{~cm}^{2}$ solid water (Constanitinou et al 1982) slab phantom was used. The radiochromic films used were Gafchromic HS Lot no I0144HS, Gafchromic MD-55-2 Lot no 37350 and MD-55-1 (Lot no Unknown). The films were irradiated up to doses of 10 Gy in 1 Gy intervals. Precautions in handling of radiochromic film outlined in TG-55 (Niroomand-Rad et al 1998) were used. The film during experiment, storage and analysis were kept in temperatures of $22^{\circ} \mathrm{C} \pm 2{ }^{\circ} \mathrm{C}$ thus reducing the effects of time- and temperaturedependent evolution and readout (Meigooni et al 1996) of the absorption spectra of the film. The film is only removed from a light tight envelope during irradiation and readout to reduce the effects of ambient light (Butson et al 1998). The absorption spectra and sensitivity to dose of the radiochromic film was measured using a Shimadzu UV-160 UV-visible recording spectrophotometer in the wavelength range of $400-800 \mathrm{~nm}$. The spectral bandwidth is $3 \mathrm{~nm}$, the wavelength readout $\pm 0.1 \mathrm{~nm}$, and has a wavelength accuracy of $\pm 0.5 \mathrm{~nm}$. It uses a double beam system and can measure optical density (OD) within 0.005 OD units. Dose comparisons were also made using Kodak EDR-2 radiographic film. This film was also irradiated to $10 \mathrm{~Gy}$ in 1 Gy intervals. 


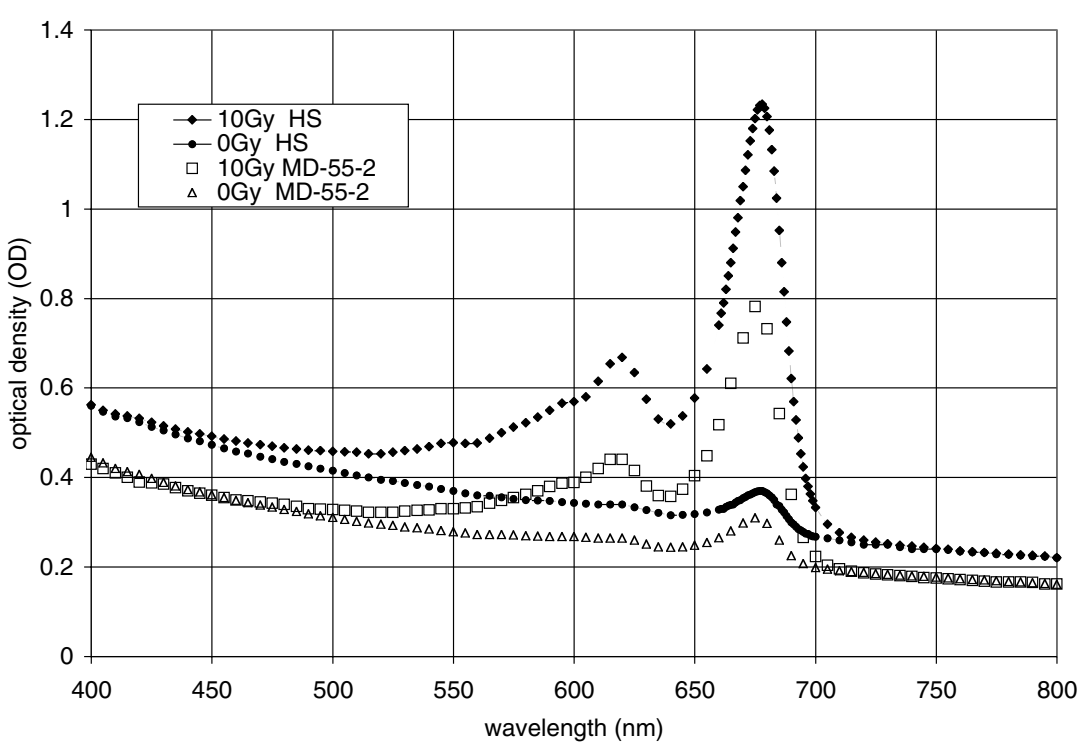

(a)

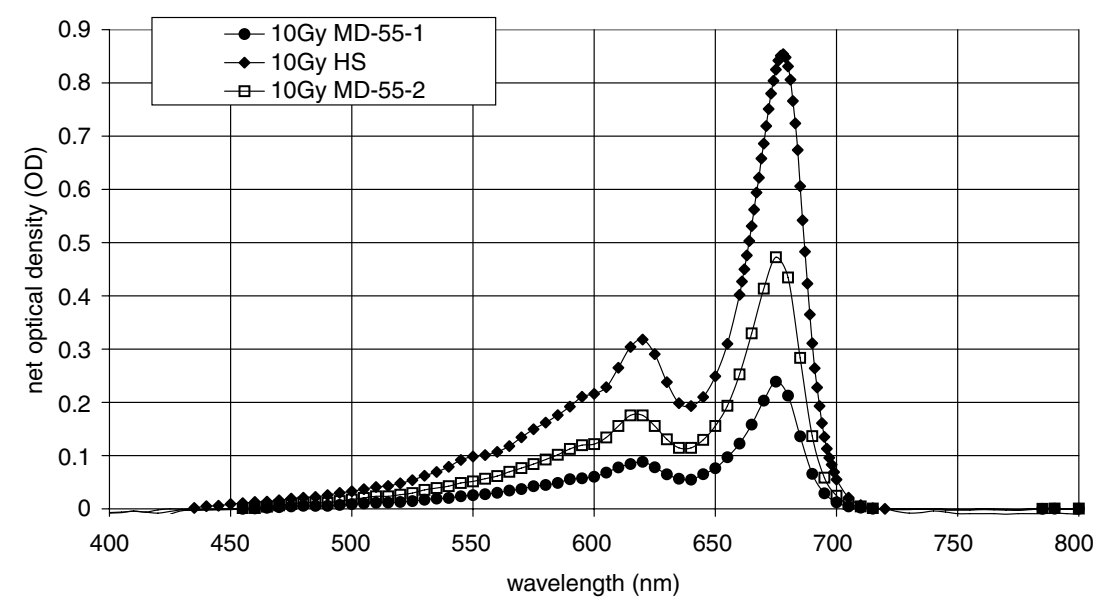

(b)

Figure 2. (a) Absolute optical density absorption spectra for HS and MD-55-2 Gafchromic film. Doses applied are 0 Gy and $10 \mathrm{~Gy}$. (b) Net optical density absorption spectra for HS, MD-55-2 and MD-55-1 Gafchromic film irradiated to 10 Gy applied dose.

\section{Results and discussion}

Figure 1 shows the energy dependence of the Gafchromic films tested in the low energy range. The results are normalized to 1 for a $6 \mathrm{MV}$ linear accelerator beam. The three energy dependences are relatively the same within error measurements. The active layer used in all three films is quoted as the same material by the manufacturer. The substrates and binding materials are different for each film, however, results show that these materials do not significantly affect the energy response of the film. At $28 \mathrm{keV}$ effective energy the response for HS film is approximately 0.64 increasing to 0.75 at $39 \mathrm{keV}$. These values produce 


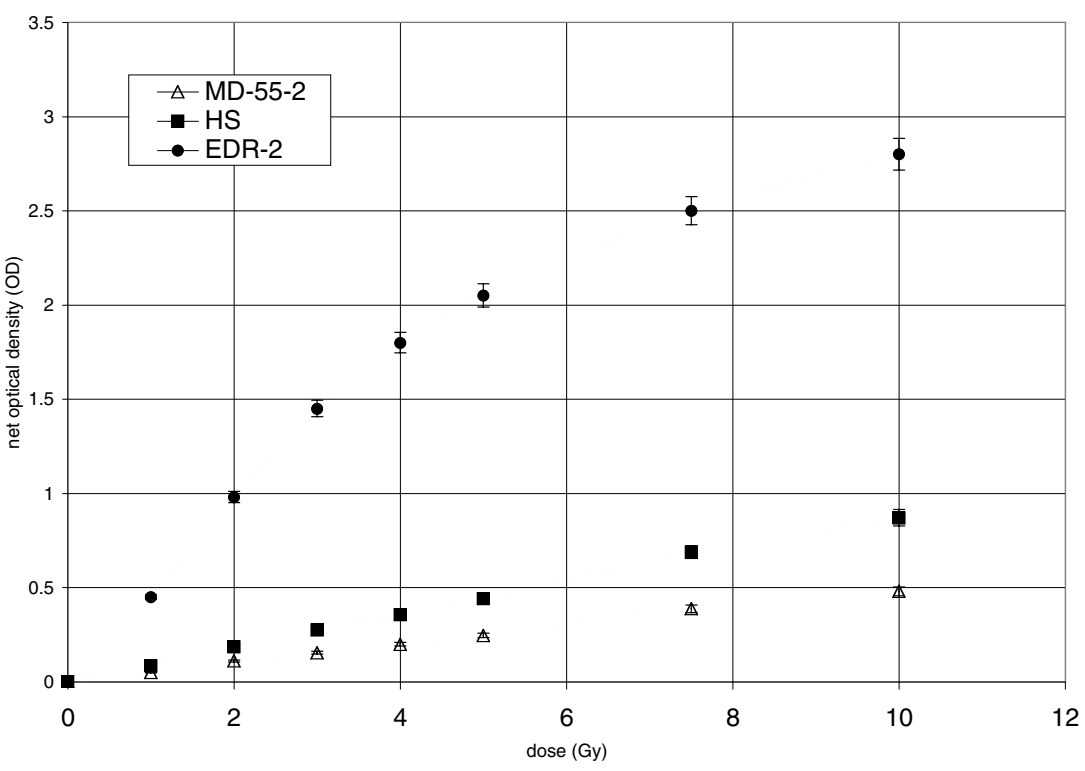

Figure 3. Dose response characteristics for HS and MD-55-2 Gafchromic film compared to EDR-2 radiographic film.

a significantly smaller energy effect than EDR-2 which was measured to have a normalized dose response of 9.2 at $28 \mathrm{keV}, 10.5$ at $32.5 \mathrm{keV}, 10.3$ at $39 \mathrm{keV}, 8.3$ at $53 \mathrm{keV}, 4.8$ at $68 \mathrm{keV}$, 4.3 at $94 \mathrm{keV}$ and 2.8 at $119 \mathrm{keV}$ ( 1 at $6 \mathrm{MV}$ ). This factor may be important for the measurement of dose in IMRT applications near multileaf collimator jaws/penumbral regions where the effective linear accelerator $\mathrm{x}$-ray spectrum comprises a larger component of low energy photons compared to the central axis position.

Figure 2(a) shows the absorption spectrum (absolute optical density) over the wavelength range of $400 \mathrm{~nm}$ to $800 \mathrm{~nm}$ for HS and MD-55-2 film. Results for an unirradiated film and 10 Gy applied dose are shown. The HS film produces an increased sensitivity over all the wavelengths which is highlighted at the absorption peak. Figure 2(b) shows the net optical density for the HS, MD-55-2 and the MD-55-1 film irradiated to a dose of 10 Gy. These results are the subtraction of absolute OD from an unirradiated piece of film from the irradiated film. Initial film OD variations account for the small negative net ODs recorded at wavelengths around $400 \mathrm{~nm}$ and $750-800 \mathrm{~nm}$. The HS film produces a sensitivity increase of approximately 1.9 times above that of the MD-55-2 film and approximately 3.8 times that of the original MD-55-1 film at $675 \mathrm{~nm}$ wavelength.

Figure 3 shows a dose sensitivity comparison of HS film to MD-55-2 and EDR-2. The net OD readings were taken at $675 \mathrm{~nm}$ for all films. Results show that the HS produces a dose sensitivity which is approximately 20-25\% that of EDR-2. The relatively high sensitivity output for HS film along with its relatively low energy dependence means that the film could be advantageous for measurement of doses in IMRT applications or any radiotherapy applications where a variation in energy spectra produced by the linac occurs.

\section{Conclusion}

Gafchromic HS film produces a higher dose response than its predecessors with a relatively unaffected energy response. That is, lower response for low energy down to 0.64 at $28 \mathrm{keV}$ 
effective energy. Its dose response is approximately 20-25\% that of EDR-2 film but produces a much better energy response (EDR-2 gives an over response of 9.2 at $28 \mathrm{keV}$ ) which may prove useful in regions of $\mathrm{x}$-ray spectral ambiguity such as penumbral regions or near MLC jaws.

\section{Acknowledgment}

This work has been fully supported by a grant from the Research Grants Council of HKSAR, China (Project No CityU 1012/01P).

\section{References}

Butson M J, Mathur J and Metcalfe P E 1995 Dose characteristics of a new 300 kVp orthovoltage machine Australas. Phys. Eng. Sci. Med. 18 133-8

Butson M, Yu P and Metcalfe P 1998 Effects of readout light sources and ambient light on radiochromic film Phys. Med. Biol. 43 2407-12

Constanitinou C, Attix F and Paliwal B 1982 A solid water phantom material for radiotherapy x-ray and gamma ray beam ray calculations Med. Phys. 9 436-41

Klassen N, Zwan L and Cygler J 1997 Gafchromic MD-55: investigated as a precision dosimeter Med. Phys. 24 1924-34

McLaughlin W, Yun-Dong C, Soares C, Miller A, Dyk G and Lewis D 1991 Sensitometry of the response of a new radiochromic film dosimeter to gamma radiation and electron beams Nucl. Instrum. Methods Phys. Res. A $\mathbf{3 0 2}$ $165-76$

Meigooni A, Sanders M, Ibbott G and Szeglin S 1996 Dosimetric characteristics of an improved radiochromic film Med. Phys. 23 1883-8

Niroomand-Rad A, Blackwell C, Coursey B, Gall K, Galvin J, McLaughlin W, Meigooni A, Nath R, Rodgers J and Soares C 1998 Radiochromic film dosimetry: recommendation of AAPM radiation therapy task group $55 \mathrm{Med}$. Phys. 25 2093-115 